\title{
Therapeutic and protective effects of autologous serum in amikacin-induced ototoxicity
}

\author{
I B ARSLAN ${ }^{1}$, G G ASLAN $^{1}$, G C MERCAN $^{1}$, S VATANSEVER ${ }^{2}$, I CUKUROVA ${ }^{1}$, \\ $\mathrm{S} \mathrm{GOKALP}^{2}$, A ASLAN ${ }^{3}$ \\ ${ }^{1}$ ENT Clinic, Tepecik Research and Training Hospital, Izmir, and Departments of ${ }^{2}$ Histology, and ${ }^{3}$ ORL, Celal \\ Bayar University School of Medicine, Manisa, Turkey
}

\begin{abstract}
Objective: Possible therapeutic and protective benefits of intratympanic autologous serum application in amikacininduced ototoxicity were investigated.

Methods: Twenty-four guinea pigs were separated equally into two groups: therapeutic (group A) and protective (group B). Transient evoked otoacoustic emissions were recorded before and after autologous serum application. Apoptotic cells were identified in the organ of Corti, spiral limbus and spiral ganglion by the terminal deoxynucleotidyl transferase-mediated dUTP nick-end labelling ('TUNEL') method.

Results: Transient evoked otoacoustic emission responses at $1,1.4$ and $2.8 \mathrm{kHz}$ improved without significance after autologous serum application in group A $(p>0.05)$. A significantly protective effect of autologous serum was determined at $4 \mathrm{kHz}$ in group B $(p<0.05)$. There were significantly fewer apoptotic cells at the spiral limbus in the therapeutic and protective groups compared to the control group $(p<0.05)$.

Conclusion: Autologous serum may offer protection against ototoxicity-induced hearing loss, but it cannot restore hearing. Immunohistochemically, autologous serum significantly decreases activation of the intrinsic pathway of pro-apoptotic signalling in mesenchymal cells compared to neurons and neurosensory cells.
\end{abstract}

Key words: Autologous Serum; Ototoxicity; Apoptosis; Organ Of Corti; Spiral Limbus; Ganglion Cell

\section{Introduction}

Ototoxicity is a severe side effect of aminoglycoside antibiotics and may result in sensorineural hearing loss. This hearing loss may be permanent and bilateral, and there is no specific therapy for it. ${ }^{1,2}$ Histological studies have revealed that aminoglycoside-induced ototoxicity begins in the outer hair cells at the basal portion of the cochlea and subsequently affects the inner hair cells. The degeneration of nerve fibres, spiral ganglion neurons and supporting cells is secondary to hair cell damage. ${ }^{3,4}$ Many reports have discussed possible prevention methods for ototoxicity, such as anti-inflammatory drugs, antioxidants, apoptosis inhibitors, neuroprotective compounds, neurotrophic factors and gene therapeutic approaches. ${ }^{2,5,6}$ In recent decades, stem cells have also been used to replace deficient cell types in order to restore hearing. ${ }^{7}$

Serum is a complex mixture of factors and molecules involved in cell growth. Growth factors are biologically active polypeptides produced by the body that can stimulate cell division, growth and differentiation. ${ }^{8}$ In addition, healing factors such as nerve growth factor, epidermal growth factor, transforming growth factor $\beta$, platelet-derived growth factors and insulin-like growth factor-1 are present in both diluted and undiluted serum. ${ }^{9}$ Nerve growth factor is the bestknown neurotrophic factor; it is responsible for the growth and survival of developing neurons, and the maintenance of mature neurons. ${ }^{10,11}$ Recent studies have indicated the efficacy of autologous serum in the treatment of neurotrophic keratopathy, osteoarthritis, chronic graft-versus-host disease and tympanic membrane perforations. ${ }^{11-13}$ In addition to treatment, autologous serum should be able to prevent or at least decrease cell damage via its anti-apoptotic factors. ${ }^{14}$ It is these potentially beneficial substances that may provide medical benefits to diseased or damaged inner-ear cells and nerves, in a therapeutic and protective manner. ${ }^{11,15}$

Apoptosis can be induced in cells by application of aminoglycosides. The terminal deoxynucleotidyl transferase-mediated dUTP (2́-deoxyuridine, 5-triphosphate) nick-end labelling ('TUNEL') technique has been extensively used in the detection and quantification of 
apoptosis in histological tissue sections. ${ }^{16}$ In addition, transient evoked otoacoustic emissions (TEOAEs) provide an important and useful electrophysiological technique for monitoring ototoxicity by reflecting changes in the functions of outer hair cells damaged by aminoglycosides, particularly in animal experiments. ${ }^{6,17}$

In this study, we hypothesised that autologous serum would have beneficial therapeutic and/or protective effects on ototoxicity generated by amikacin in guinea pigs. Its efficacy was evaluated by TEOAE and immunohistopathological examination.

\section{Materials and methods}

This study was approved by the Ethical Committee for Animal Experimentation at Celal Bayar University, Manisa, Turkey (reference number: 77.637.435-14). All surgical procedures were completed in accordance with the National Institutes of Health guidelines on the care and use of laboratory animals for research purposes.

In total, 24 female albino guinea pigs, with body weights ranging from 700 to $900 \mathrm{~g}$, were used in the study. These were equally separated into a therapeutic group (group A) and a protective group (group B), with positive and negative controls created within each group. Only the right ears of the guinea pigs were used.

Transient evoked otoacoustic emissions (TEOAEs) were recorded using an Echoport ILO292 USB-I device with ILO version 5 software (Otodynamics, Hatfield, Herts, UK). The TEOAEs were evoked using clicks of $63 \pm 2 \mathrm{~dB}$ at a rate of 50 per second, with a click duration of $80 \mu \mathrm{s}$. Response frequency bands were in half octaves, centred at 1, 1.4, 2, 2.8 and $4 \mathrm{kHz}$. Signal-to-noise ratio is the amplitude of otoacoustic emissions above the noise floor and is measured in decibels of sound pressure levels; signal-tonoise ratio was recorded at these centre frequencies. Reproducibility and probe stability percentage were also recorded. Reproducibility was measured as the percentage correlation between two averages during data collection. Stability indicates the stability of the stimulus peak level during the otoacoustic emission recording. It shows the stability of the probe fit during measurement. The stability of the response is high if all the stimuli remain almost the same throughout the test.

Autologous serum was collected from the lateral saphenous vein, under anaesthesia with intraperitoneal injections of xylazine hydrochloride $(10 \mathrm{mg} / \mathrm{ml})$ (Bioveta, Ivanovice na Hané, Czech Republic) and ketamine $(50 \mathrm{mg} / \mathrm{ml})$ (Pfizer, Istanbul, Turkey). Blood samples were centrifuged for 5 minutes at 3000 revolutions per minute. ${ }^{13}$ The resulting serum was placed in a dental syringe and stored at $4^{\circ} \mathrm{C}$. In order to create ototoxicity, amikacin (Amikozit; EczacibasiZentiva, Istanbul, Turkey) was administered at a dose of $0.2 \mathrm{cc}(80 \mathrm{mg} / \mathrm{ml})$ via the intratympanic and intramuscular routes, respectively.

\section{Therapeutic group}

Prior to the procedure, the TEOAE responses of the guinea pigs in group A were recorded. In the first week, eight guinea pigs (the study group) received $0.2 \mathrm{cc}$ of intratympanic amikacin three times to generate hearing loss. The positive control group of two guinea pigs received $0.2 \mathrm{cc}$ of amikacin; the negative control group, also composed of two guinea pigs, received $0.2 \mathrm{cc}$ of saline. The TEOAE responses were evaluated in both the study and positive control groups after amikacin application. In the second week, $0.2 \mathrm{cc}$ of autologous serum was administered three times to the study group, beginning after the 3 days of amikacin application. In the third week of the study, the TEOAE responses of the guinea pigs were obtained and the animals were sacrificed. The guinea pig cochleae were removed for immunohistochemical examination.

\section{Protective group}

The TEOAE responses of the guinea pigs in group B were obtained initially. Ten guinea pigs (eight from the study group and two from the positive control group) received $0.2 \mathrm{cc}$ of amikacin intramuscularly every day of the week. Two guinea pigs (the negative control group) received $0.2 \mathrm{cc}$ of 0.9 per cent saline. Autologous serum $(0.2 \mathrm{cc})$ was applied intratympanically three times in a week, at the same time as the intramuscular amikacin administration. One week after the last autologous serum application, the TEOAE responses of the guinea pigs were recorded and the animals were sacrificed for immunohistochemical examination.

\section{Histopathology}

Cochlear samples were fixed in 10 per cent formalin for 24-48 hours and then embedded in paraffin blocks after routine processing. A rotary microtome was used to cut the samples into $5 \mu \mathrm{m}$ sections. Haematoxylin and eosin staining was used to examine the tissue morphology using standard protocols. Apoptotic cells in the tissue were determined using the terminal deoxynucleotidyl transferasemediated dUTP nick-end labelling method.

\section{Terminal deoxynucleotidyl transferase-mediated dUTP nick-end labelling assay}

The paraffin sections were rehydrated after deparaffinisation in xylene. After washing with phosphatebuffered saline, they were incubated with $20 \mu \mathrm{g} / \mathrm{ml}$ of proteinase $\mathrm{K}$ for 10 minutes. The sections were then incubated with 3 per cent hydrogen peroxide for 15 minutes. After adding equilibration buffer for 10-15 minutes, terminal deoxynucleotidyl transferase enzyme was added and the sections were incubated in a humidified atmosphere at $37^{\circ} \mathrm{C}$ for 60 minutes. The slides were washed with stop/wash buffer at room temperature for 10 minutes. Streptavidin- 
peroxidase solution was then added and the slides were incubated for 45 minutes at room temperature. After each step, the slides were washed with phosphate-buffered saline.

Staining was completed with diaminobenzidine, and counterstaining was performed with Mayer's haematoxylin (HMM999; Scytek Laboratories, Logan, Utah, USA). The in situ apoptosis detection kit (S7100; Invitrogen, New York, USA) was used for this assay. After mounting with Entellan ${ }^{\circledR}$ medium, the slides were examined independently by two histologists; 100 cells which were brown-stained were accepted as terminal deoxynucleotidyl transferasemediated dUTP nick-end labelling positive in each organ of Corti, spiral limbus and ganglion on randomly chosen fields per case. The percentage of terminal deoxynucleotidyl transferase-mediated dUTP nickend labelling positive cells which were stained among 100 cells was determined. ${ }^{18}$

\section{Immunohistochemistry}

The paraffin-embedded sections were washed with phosphate-buffered saline for 15 minutes. Endogenous peroxidase activity was quenched by incubation with 3 per cent hydrogen peroxide (K31355100; Merck, Darmstadt, Germany) for 5 minutes at room temperature. Cells were then washed with phosphate-buffered saline, and incubated in blocking serum (ready to use according to the manufacturer's instruction) (85-9043; Invitrogen, Camarillo, California, USA) for 1 hour.

The primary antibodies anti-Bax (DB005; Delta Biolabs, Gilroy, California, USA), anti-caspase 3 (ab2302; Abcam, Cambridge, UK), anti-cytochrome c (sc-13156; Santa Cruz Biotechnology, Dallas, Texas, USA) and anti-Fas ligand (FasL-DB042; Delta Biolabs) were applied at $4{ }^{\circ} \mathrm{C}$ overnight in a dilution of 1:100. After removal of the primary antibodies, the slides were washed with phosphate-buffered saline. The secondary antibodies biotinylated goat immunoglobulin G (IgG) anti-rabbit/mouse IgG and peroxidase-conjugated streptavidin (D01-110; Life Science Research, Merck, Darmstadt, Germany) were applied, and the cells were incubated for 30 minutes for each step.

Between the secondary antibody incubation steps and after peroxidase-conjugated streptavidin, the cells were washed with phosphate-buffered saline three times. The samples were then incubated with diaminobenzidine/hydrogen peroxide (00-2020; Invitrogen, Camarillo, California, USA) for 5 minutes. Cells were counterstained with Mayer's haematoxylin (HMM999; Scytek Laboratories).

After washing with distilled water, the cells were mounted with mounting medium (AML060; Scytek Laboratories) and covered with coverslips before evaluation under a light microscope (Olympus BX40; Olympus, Tokyo, Japan).

The negative control samples were processed in an identical manner, but instead of primary antibodies the same type of IgGs were used. ${ }^{18}$

\section{Evaluation of sections}

Immunolabelling intensity was graded (independently, by two observers who were blinded to the experimental conditions) as follows: weak, moderate and strong.

\section{Statistical analysis}

Statistical analysis was performed with SPSS software, version 20.0 for Windows (IBM, Armonk, New York, USA). The study data are expressed as mean values. For the immunohistochemical analysis, statistical comparisons of continuous variables among the groups were performed using a Kruskal-Wallis test based on their abnormal distribution. A paired samples $t$-test was performed for the analysis of TEOAE responses and apoptotic cells. A $p$-value of less than 0.05 was considered to be statistically significant.

\section{Results}

One guinea pig in the therapeutic group died before sacrifice. After amikacin administration, the signal-to-noise ratio from the TEOAE recordings of both the study and positive control groups in the therapeutic group (group A) were significantly decreased for all frequencies $(p<0.05)$. The signal-to-noise ratio at $1,1.4$ and $2.8 \mathrm{~Hz}$ were found to be slightly increased after autologous serum application, but without significance $(p>0.05)$. The TEOAE responses for all frequencies decreased after the intramuscular administration of amikacin in the protective group (group B). A significantly protective effect of autologous serum was observed only at $4 \mathrm{kHz}(p<0.05)$ (Table I).

No therapeutic or protective effects of autologous serum were observed in the organ of Corti according to our immunohistochemical analysis following autologous serum administration $(p>0.05)$. Both the therapeutic and protective groups' apoptotic cells in the spiral limbus were significantly reduced compared to the positive control group $(p<0.05)$. Apoptosis was reduced in the spiral ganglion after autologous serum application compared to the positive controls in groups A and $\mathrm{B}$, but without significance $(p>0.05)$ (Table II and Figure 1).

When the mean number of apoptotic cells of the guinea pigs in both study groups were compared with the mean value of the positive control groups, a significantly protective effect was observed in four spiral limbus and in three spiral ganglion samples from the animals in group B (Table III).

The immunoreactivity of Bax and Casp3 was greater than that of sit-C and Fas ligand, suggesting that the intrinsic mitochondrial pathway was primarily activated. The weak immune reaction of Fas ligand indicates that the extrinsic pathway was not triggered as much in this case. All apoptotic pathways, and particularly the intrinsic pathway, were more affected in the positive control groups than in the study and negative control groups (Table IV). 


\begin{tabular}{|c|c|c|c|c|c|c|c|}
\hline \multirow{3}{*}{ Group } & \multicolumn{5}{|c|}{ TABLE I } & \multirow{3}{*}{ Reproducibility $^{\dagger}(\%)$} & \multirow{3}{*}{ Stability $^{\ddagger}(\%)$} \\
\hline & \multicolumn{5}{|c|}{ Signal-to-noise ratio* (dB SPL) } & & \\
\hline & $1 \mathrm{kHz}$ & $1.4 \mathrm{kHz}$ & $2 \mathrm{kHz}$ & $2.8 \mathrm{kHz}$ & $4 \mathrm{kHz}$ & & \\
\hline \multicolumn{8}{|l|}{ Therapeutic } \\
\hline - Before amikacin & 5.22 & 6.98 & 12.61 & 15.61 & 8.72 & 89 & 99 \\
\hline - After amikacin & -4.1 & -5.82 & -7.76 & -4.82 & -5.42 & 78 & 98.8 \\
\hline - After autologous serum & -4.08 & 0.10 & -10.36 & -3.1 & -8.24 & 80 & 98.4 \\
\hline \multicolumn{8}{|l|}{ Protective } \\
\hline - Before amikacin & 10.02 & 12.79 & 14.64 & 13.64 & 12.59 & 87 & 95 \\
\hline - After amikacin & 0.64 & 1.68 & 3.57 & 3.17 & 6.45 & 75 & 97 \\
\hline \multicolumn{8}{|l|}{ Therapeutic positive control } \\
\hline - Before amikacin & 6.88 & 8.94 & 12.46 & 14.72 & 10.45 & 90 & 98 \\
\hline - After amikacin & -2.8 & -8.8 & -6.3 & -3.9 & -4.7 & 85 & 93 \\
\hline \multicolumn{8}{|l|}{ Protective positive control } \\
\hline - Before amikacin & 3.9 & 7.2 & 15.4 & 16.4 & 11.2 & 94 & 97 \\
\hline - After amikacin & -1.8 & -0.8 & 1.8 & 4.5 & 2.1 & 87 & 96 \\
\hline
\end{tabular}

*Indicates the amplitude of otoacoustic emissions above the noise floor. ${ }^{\dagger}$ Indicates the percentage correlation between two averages during data collection for otoacoustic emission measurement. ${ }^{\ddagger}$ Stability of the stimulus peak level during the otoacoustic emission recording, reflecting the stability of the probe fit during measurement. TEOAEs $=$ transient evoked otoacoustic emissions

\section{Discussion}

The protection of both hair cells and neurons is a highly attractive strategy for combating ototoxicity. In order to prevent ototoxic cell death, researchers have developed various strategies, including the inhibition of apoptosis, the neutralisation of reactive oxygen species and the administration of neurotrophic factors. Anti-apoptotic agents confer significant protection against hair cell damage from aminoglycosides, preserving hair cell morphology and function in vitro and in vivo. ${ }^{19-21}$ Unfortunately, long-term treatment with anti-apoptotic agents is associated with a potential carcinogenic risk because apoptosis plays a crucial primary role in preventing uncontrolled cell proliferation. ${ }^{22}$

A large number of studies have investigated the otoprotective effects of antioxidants (e.g. aspirin, $\mathrm{N}$-acetylcysteine, dihydroxybenzoate and melatonin) on aminoglycoside ototoxicity. Overall, antioxidants attenuate ototoxic damage from aminoglycosides. However, the majority of antioxidants have not demonstrated complete protection against aminoglycoside ototoxicity. ${ }^{23,24}$ The contribution of neurotrophic growth factors in preventing aminoglycoside ototoxicity suggests involvement of the auditory nerve and sensorineural cells. However, evidence exists which indicates that the effects of neurotrophic growth factors alone are not beneficial. ${ }^{25}$

Autologous serum can be considered a good candidate for ototoxicity prevention. Because autologous serum incorporates anti-apoptotic activity, it is able to increase the antioxidant capacity of tissues and it contains growth factors. ${ }^{26-28}$ Autologous serum has been used over the last two decades to promote the healing of epithelial and cartilaginous damage of both known and unknown aetiologies. ${ }^{13,29-31}$ At the same time, it includes various immunoglobulins (e.g. immunoglobulin $\mathrm{G}$ and immunoglobulin A) and lysozyme. , $32^{8,2}$

Apoptosis is a distinct form of cell death controlled by an internally encoded suicide programme that includes independent extrinsic and intrinsic pathways. Amikacin, which is not metabolised and remains in hair cells for months, causes the formation of reactive oxygen species, leading to apoptosis. When the formation of reactive oxygen species overwhelms the capacity of the intrinsic protective and repair systems, the cell undergoes apoptotic cell death, resulting in ototoxicity and deafness. ${ }^{3,33,34}$

The present study evaluated the possible therapeutic and protective effects of autologous serum on amikacin-induced apoptosis in an animal model, by both

\begin{tabular}{|c|c|c|c|c|c|}
\hline \multicolumn{6}{|c|}{ TABLE II } \\
\hline Group & Division & Study group & Positive control group & $P$-value* & Negative control group \\
\hline \multirow[t]{3}{*}{ Therapeutic } & Organ of Corti & 46.6 & 47.27 & 0.87 & 7.53 \\
\hline & Spiral limbus & 42.5 & 82.18 & 0.01 & 23.5 \\
\hline & Ganglion & 40.29 & 41.25 & 0.72 & 24 \\
\hline \multirow[t]{3}{*}{ Protective } & Organ of Corti & 40.6 & 46.18 & 0.41 & 7.06 \\
\hline & Spiral limbus & 61.12 & 79.75 & 0.04 & 7.66 \\
\hline & Ganglion & 40.77 & 57.92 & 0.49 & 6.77 \\
\hline
\end{tabular}

Data represent mean apoptotic cell count, unless indicated otherwise. ${ }^{*} P$-values concern the differences between the study group and the positive control group. 

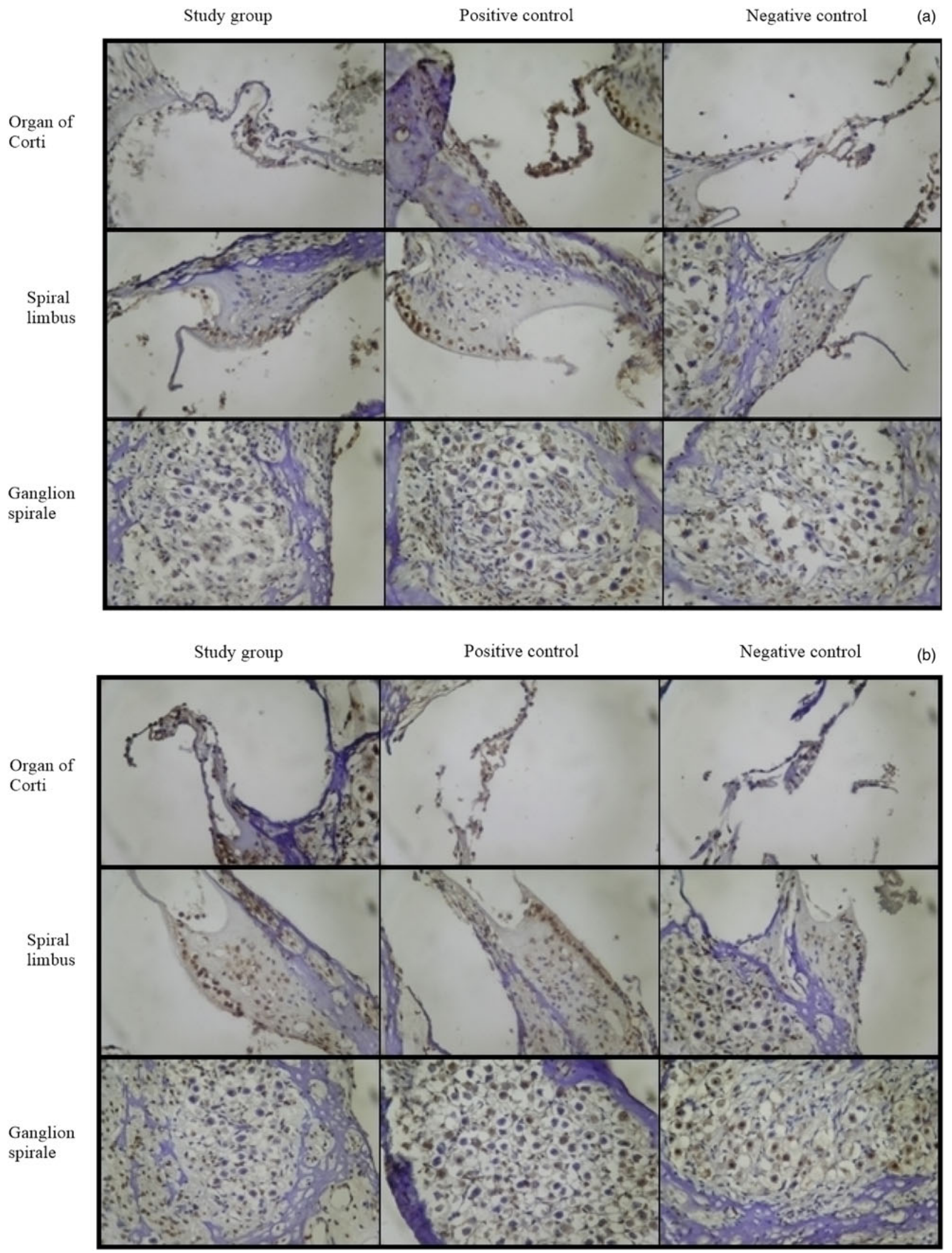

FIG. 1

Immunohistochemical examination of (a) therapeutic group (group A) and (b) protective group (group B), for both study and control groups. $(\mathrm{H} \& \mathrm{E} ; \times 200)$ 


\begin{tabular}{lcccc}
\multicolumn{5}{c}{ TABLE III } \\
COMPARISON BETWEEN APOPTOTIC CELL COUNT OF \\
EACH INDIVIDUAL ANIMAL AND MEAN VALUE OF \\
POSITIVE CONTROL GROUP ANIMALS \\
\hline Group & $\begin{array}{c}P- \\
\text { value }\end{array}$ & $\begin{array}{c}\text { Organ of } \\
\text { Corti }\end{array}$ & $\begin{array}{c}\text { Spiral } \\
\text { limbus }\end{array}$ & Ganglion \\
& $<0.05$ & 0 & 0 & 0 \\
Therapeutic & $>0.05$ & 7 & 7 & 7 \\
Protective & $<0.05$ & 8 & 4 & 3 \\
& $>0.05$ & 0 & 4 & 5 \\
\hline
\end{tabular}

Data represent mean apoptotic cell count, unless indicated otherwise.

TEOAE and histopathological examination. After amikacin application, TEOAE assessments revealed a statistically significant decrease in signal-to-noise ratio in group A. Autologous serum had no therapeutic effect on hearing function according to the TEOAE responses, but its significantly protective effects appeared at $4.0 \mathrm{kHz}$. However, intratympanic amikacin may have reached a higher concentration in the inner ear. Thus, intratympanic amikacin is more destructive than when it is administered intramuscularly. In addition to this, autologous serum might be effective in partial or minor cell destruction according to the TEOAE responses. The number of apoptotic cells was significantly higher in the negative control guinea pigs that received intratympanic rather than intramuscular administration of 0.9 per cent saline $(p<0.05)$.

The main purpose of autologous serum application is to induce healing via the complex autologous serum mixture, which harbours growth factors, vitamins and immunoglobulins. Recently, the local application of growth factors to the inner ear has been presented as an effective method for protecting the cochlea from insertional trauma during cochlear implantation. ${ }^{35}$ Autologous serum may inhibit apoptotic cascades or activate healing factors via multiple endocytic mechanisms in neuro-sensitive, mesenchymal and neural cells. On the other hand, serum components such as lysozyme may have a detrimental effect on inner-ear cells. Inflammation and lysozyme may trigger apoptosis in the rather sensitive cells of the organ of Corti. ${ }^{8}$ The decreased therapeutic and protective effects of autologous serum in the organ of Corti compared to the spiral limbus and ganglion cells may be explained by lysozyme activity. Autologous serum lacking lysozyme, which has a destructive effect on cells, may improve the healing and protective effects of autologous serum.

The histopathological results of aminoglycoside ototoxicity revealed that nerve fibres, spiral ganglion neurons and supporting cells were damaged much later than hair cells. Strial degeneration has been reported previously, but the authors were unsure whether it was a secondary or primary effect of aminoglycoside treatment. ${ }^{4,33}$ The connective tissue cells of the spiral limbus are highly susceptible to ototoxicity. At the same time, the spiral limbus may serve as one of the most sensitive indicators of changes in the chemical composition of perilymph. ${ }^{36}$ The highest apoptotic cell count was encountered in the spiral limbus of both positive control groups when compared with ganglion cells and the organ of Corti. According to the present study, the spiral limbus is more susceptible to amikacin-induced ototoxicity than ganglion cells and the organ of Corti.

It is thought that mammalian cochlear hair cells and spiral ganglion neurons are terminally differentiated, and once completely damaged they do not regenerate. ${ }^{4}$ Recently, the beneficial effect of platelet-rich plasma and neural-induced human mesenchymal stem cells was demonstrated on axonal regeneration from a facial nerve axotomy injury in a guinea pig model. ${ }^{37}$ Although a significantly protective effect was observed in three guinea pig ganglion cells, according to immunohistochemical examination, the current study did not show therapeutic or protective effects of autologous serum in ganglion cells. However, significant therapeutic and protective effects of autologous serum were detected in the spiral limbus. The current study suggests that autologous serum is beneficial in connective tissue cells like the spiral limbus, rather

TABLE IV

IMMUNOHISTOCHEMICAL ANALYSIS OF THERAPEUTIC AND PROTECTIVE GROUPS

\begin{tabular}{|c|c|c|c|c|c|c|c|c|c|c|c|c|}
\hline \multirow[t]{3}{*}{ Group } & \multicolumn{12}{|c|}{ Apoptotic pathway } \\
\hline & \multicolumn{4}{|c|}{ Organ of Corti } & \multicolumn{4}{|c|}{ Spiral limbus } & \multicolumn{4}{|c|}{ Ganglion cell } \\
\hline & Bax & Sit-C & Casp3 & Fas ligand & Bax & Sit-C & Casp3 & Fas ligand & Bax & Sit-C & Casp3 & Fas ligand \\
\hline \multicolumn{13}{|l|}{ Therapeutic } \\
\hline - Study & ++ & + & + & + & + & + & + & + & +++ & ++ & ++ & + \\
\hline - Positive control & ++ & + & ++ & ++ & ++ & + & ++ & + & +++ & ++ & +++ & ++ \\
\hline $\begin{array}{l}\text { - Negative control } \\
\text { Protective }\end{array}$ & + & 0 & + & + & + & 0 & + & + & + & + & + & + \\
\hline - Study & ++ & + & + & + & + & + & + & + & +++ & + & ++ & + \\
\hline - Positive control & ++ & ++ & ++ & + & ++ & + & ++ & + & +++ & + & ++ & + \\
\hline - Negative control & + & + & + & + & + & 0 & 0 & 0 & + & + & 0 & 0 \\
\hline
\end{tabular}

Immunolabelling intensity: ' 0 ' $=$ absent, ' + ' = weak, ' $++{ }^{\prime}=$ moderate and ' +++ ' $=$ strong 
than in sensorineural and neural cells. Our immunohistochemical examination revealed that the intrinsic mitochondrial pathway, particularly Bax and Casp3, was activated to a greater extent than the extrinsic pathway.

\section{- Autologous serum is a new treatment option} which harbours growth factors, vitamins and immunoglobulins

- Ototoxic cell death can be induced by apoptosis inhibition, reactive oxygen species neutralisation and neurotrophic factor administration

- Autologous serum may inhibit apoptosis or activate healing via endocytic mechanisms in neuro-sensitive, mesenchymal and neural cells

- This study indicates that autologous serum may be protective against ototoxicity-induced hearing loss

This study has a number of limitations, including a lack of low- and high-frequency thresholds in the TEOAE assessment. The lack of quality control prevented assessment of autologous serum levels among the animals. This study explored uncontrolled autologous serum levels after application. In addition, the levels of growth factors and lytic enzymes were not determined. Future research should focus on the enhancement of autologous serum with growth factors and antioxidant agents, to create a greater anti-apoptotic effect. The anti-inflammatory properties of cytokines in autologous serum should be useful in treating autoimmune inner-ear diseases.

\section{Conclusion}

Autologous serum may offer protection against ototoxicity-induced hearing loss, but it cannot restore hearing. Autologous serum conferred therapeutic and protective effects on mesenchymal cells as opposed to neurosensory or neural cells in amikacin-induced ototoxicity. However, the question of how much damage will be reversed or what amount of cell damage can be prevented remains unanswered. Further study is needed on the use of autologous serum for diseased or damaged inner-ear cells and nerves.

\section{References}

1 Shi L, An Y, Wang A, Gao Q, Yang Y. The protective effect of Salvia miltiorrhiza on gentamicin-induced ototoxicity. $\mathrm{Am} \mathrm{J}$ Otolaryngol 2014;35:171-9

2 Polony G, Humli V, Ando R, Aller M, Horvalth T, Harnos A et al. Protective effect of rasagiline in aminoglycoside ototoxicity. Neuroscience 2014;265:263-73

3 Berkiten G, Slturk Z, Topaloglu I, Ugras H. Protective effect of pentoxifylline on amikacin-induced ototoxicity in rats. $\mathrm{Am} \mathrm{J}$ Otolaryngol 2012;33:689-92

4 Guthrie OW. Aminoglycoside induced ototoxicity. Toxicology 2008;249:91-6

5 Blakley BW, Alsaleh S, Dewji Z, Qureshy K, Berard S, Xie L. Steroids in aminoglycoside-containing ear drops: do they reduce cochlear toxicity? Laryngoscope 2014;124:1209-13
6 Kocyigit I, Vural A, Unal A, Sipahioglu MH, Yucel HE, Aydemir $\mathrm{S}$ et al. Preventing amikacin related ototoxicity with $\mathrm{N}$-acetylcysteine in patients undergoing peritoneal dialysis. Eur Arch Otorhinolaryngol 2015;272:2611-20

7 Kelly KM, Lalwani AK. On the distant horizon - medical therapy for sensorineural hearing loss. Otolaryngol Clin North Am 2015;48:1149-65

8 Fortier LA, Barker JU, Strauss EJ, McCarrel TM, Cole BJ. The role of growth factors in cartilage repair. Clin Orthop Relat Res 2011;469:2706-15

9 Wehling P, Moser C, Frisbie D, McIlwraith W, Kawcak CE, Krauspe R et al. Autologous conditioned serum in the treatment of orthopedic diseases: the orthokine therapy. BioDrugs 2007; 21:323-32

10 Lang UE, Gallinat J, Danker-Hopfe H, Bajbouj M, Hellweg R. Nerve growth factor serum concentrations in healthy human volunteers: physiological variance and stability. Neurosci Lett 2003;344:13-16

11 Matsumoto Y, Dogru M, Goto E, Ohashi Y, Kojima T, Ishida R et al. Autologous serum application in the treatment of neurotrophic keratopathy. Ophthalmology 2004;11:1115-20

12 Baltzer AW, Ostapczuk MS, Stosch D, Seidel F, Granrath M. A new treatment for hip osteoarthritis: clinical evidence for the efficacy of autologous conditioned serum. Orthop Rev (Pavia) 2013:5:59-64

13 Kakehata S, Hirose Y, Kitani R, Futai K, Maruya S, Ishii K et al. Autologous serum eardrops therapy with a chitin membrane for closing tympanic membrane perforations. Otol Neurotol 2008; 29:791-5

14 Bomstein Y, Yuklea M, Radnay J, Shapiro H, Afanasyev F, Yarkoni S et al. The antiapoptotic effects of blood constituents in patients with chronic lymphocytic leukemia. Eur J Haematol 2003;70:290-5

15 Kaevalin LK, Jongkhajornpong P, Choubtum L, Chuckpaiwong V. Topical $100 \%$ serum eye drops for treating corneal epithelial defect after ocular surgery. BioMed Res Int 2013;2013:521315

16 Duan WR, Garner DS, Williams SD, Funckes-Shippy CL, Spath IS, Blomme EA. Comparison of immunohistochemistry for activated caspase-3 and cleaved cytokeratin 18 with the TUNEL method for quantification of apoptosis in histological sections of PC-3 subcutaneous xenografts. J Pathol 2003;199:221-8

17 Derinsu U, Akdas F. TEOAE findings of guinea pigs [in Turkish]. Otoskop 2003;3:127-31

18 Vatansever HS, Sorkun K, Guran SI, Ozdal-Kurt F, Turkoz E, Gencay $\mathrm{O}$ et al. Propolis from Turkey induces apoptosis through activating caspases in human breast carcinoma cell lines. Acta Histochem 2010;112:546-56

19 Forge A, Li L. Apoptotic death of hair cells in mammalian vestibular sensory epithelia. Hear Res 2000;139:97-115

20 Williams JA, Holder N. Cell turnover in neuromasts of zebrafish larvae. Hear Res 2000;143:171-81

21 Nakamagoe M, Tabuchi K, Uemaetomari I, Nishimura B, Hara A. Estradiol protects the cochlea against gentamicin ototoxicity through inhibition of the JNK pathway. Hear Res 2010;261: $67-74$

22 Olsson M, Zhivotovsky B. Caspases and cancer. Cell Death Differ 2011;18:1441-9

23 Campbell KC, Meech RP, Klemens JJ, Gerberi MT, Dyrstad SS, Larsen DL et al. Prevention of noise- and drug-induced hearing loss with D-methionine. Hear Res 2007;226:92-103

24 Erdem T, Ozturan O, Iraz M, Miman MC, Olmez E. Dosedependent dual effect of melatonin on ototoxicity induced by amikacin in adult rats. Eur Arch Otorhinolaryngol 2005;262: $314-21$

25 Gillespie LN, Clark GM, Bartlett PF, Marzella PL. BDNFinduced survival of auditory neurons in vivo: cessation of treatment leads to accelerated loss of survival effects. J Neurosci Res 2003;71:785-90

26 Quinto GG, Campos M, Behrens A. Autologous serum for ocular surface diseases. Arq Bras Oftalmol 2008;71:47-54

27 Gunay C, Sagliyan A, Yilmaz S, Kandemir FM, Han MC, Ozkaraca $\mathrm{M}$ et al. Evaluation of autologous serum eyedrops for the treatment of experimentally induced corneal alcali burns. Revue Méd Vét 2015;166:63-71

28 Poon AC, Geerling G, Dart JK, Fraenkel GE, Daniels JT. Autologous serum eyedrops for dry eyes and epithelial defects: clinical and in vitro toxicity studies. $\mathrm{Br} J$ Ophthalmol 2001;85:1188-97 
29 Kamal S, Kumar S, Goel R. Autologous serum for anterior tissue necrosis after porous orbital implant. Middle East Afr $J$ Ophthalmol 2014;21:193-5

30 Wehling P, Moser C, Frisbie D, Mcllwraith CW, Kawcak CE, Krauspe $\mathrm{R}$ et al. Autologous conditioned serum in the treatment of orthopedic diseases: the orthokine therapy. BioDrugs 2007; 21:323-32

31 Nimura A, Muneta T, Koga H, Mochizuki T, Suzuki K, Makino $\mathrm{H}$ et al. Increased proliferation of human synovial mesenchymal stem cells with autologous human serum: comparisons with bone marrow mesenchymal stem cells and with fetal bovine serum. Arthritis Rheum 2008;58:501-10

32 Geerling G, Maclennan S, Hartwig D. Autologous serum eye drops for ocular surface disorders. Br J Ophthalmol 2004;88: 1467-74

33 Huth ME, Ricci J, Cheng AG. Mechanisms of aminoglycoside ototoxicity and targets of hair cell protection. Int J Otolaryngol 2011;2011:937861

34 Alharazneh A, Luk L, Huth M, Monfared A, Steyger PS, Cheng $\mathrm{AG}$ et al. Functional hair cell mechanotransducer channels are required for aminoglycoside ototoxicity. PLoS ONE 2011;6: e22347

35 Kikkawa YS, Nakagawa T, Ying L, Tabata Y, Tsubouchi H, Ido A et al. Growth factor-eluting cochlear implant electrode: impact on residual auditory function, insertional trauma, and fibrosis. J Transl Med 2014;12:280

36 Kimura RS, Nye CL, Southard RE. Normal and pathologic features of the limbus spiralis and its functional significance. Am J Otolaryngol 1990;11:99-111

37 Cho HH, Jang S, Lee SC, Jeong HS, Park JS, Han JY et al. Effect of neural-induced mesenchymal stem cells and plateletrich plasma on facial nerve regeneration in an acute nerve injury model. Laryngoscope 2010;120:907-13

Address for correspondence:

Dr Ilker Burak Arslan,

Izmir Tepecik Egitim ve Arastirma Hastanesi KBB Klinigi, Gaziler cad. No: 468,

35170 Yenisehir,

Izmir, Turkey

Fax: +90 2324330756

E-mail: ilkerburakarslan@hotmail.com

Dr I B Arslan takes responsibility for the integrity of the content of the paper

Competing interests: None declared 